\title{
Pour une archéologie du travail savant : fiches, livres et lettres de Marcel Mauss.
}

\section{Jean-François Bert}

\section{Q OpenEdition}

1 Journals

Édition électronique

URL : https://journals.openedition.org/rbnu/2674

DOI : $10.4000 /$ rbnu. 2674

ISSN : 2679-6104

Éditeur

Bibliothèque nationale et universitaire de Strasbourg

Édition imprimée

Date de publication : 1 novembre 2012

Pagination : 18-25

ISSN : 2109-2761

\section{Référence électronique}

Jean-François Bert, «Pour une archéologie du travail savant : fiches, livres et lettres de Marcel Mauss. », La Revue de la BNU [En ligne], 6 | 2012, mis en ligne le 01 novembre 2012, consulté le 08 août 2021. URL : http://journals.openedition.org/rbnu/2674 ; DOI : https://doi.org/10.4000/rbnu.2674

\section{(c) (i) (9)}

La Revue de la BNU est mise à disposition selon les termes de la Licence Creative Commons Attribution - Pas d'Utilisation Commerciale - Partage dans les Mêmes Conditions 4.0 International. 
UToteris

Eidos

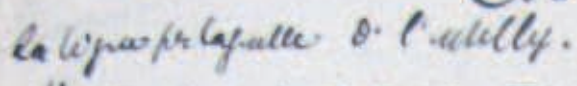

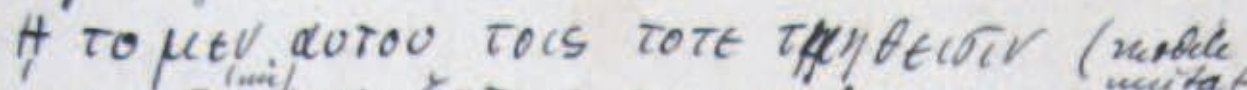

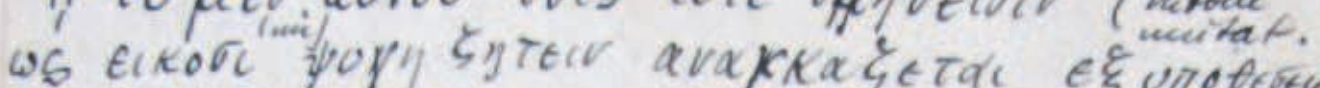

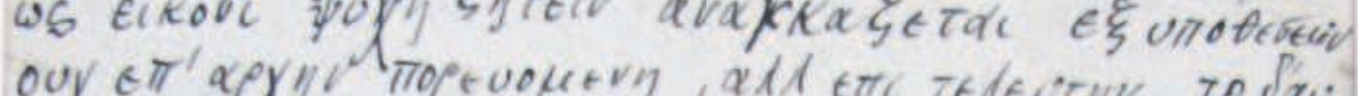
6TeO

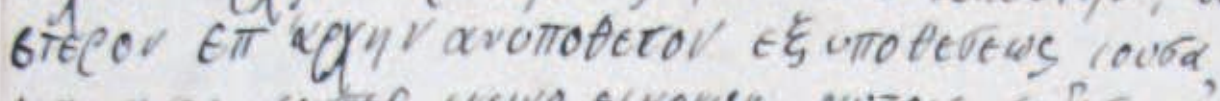

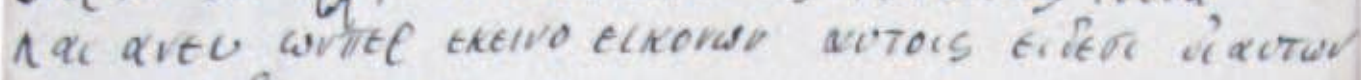
TIJ futboior mocoofetry.

(SioB)

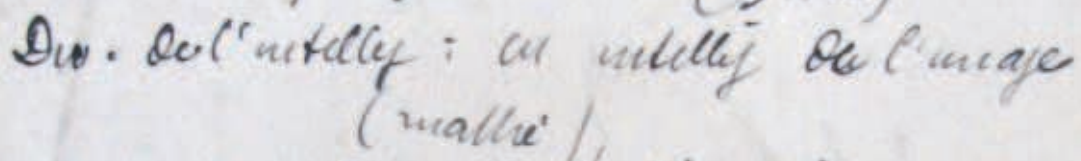

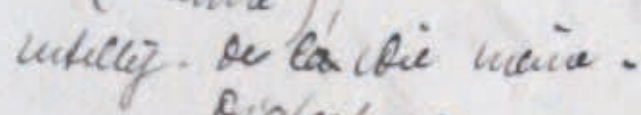

Bialeetycue

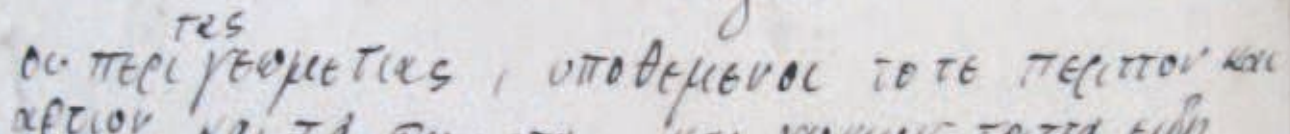

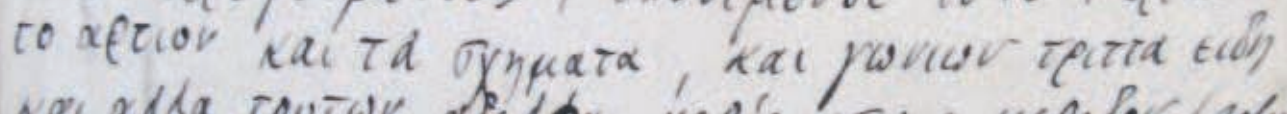

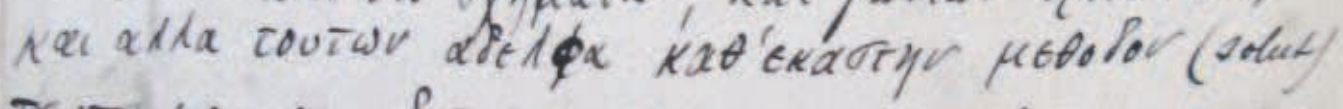

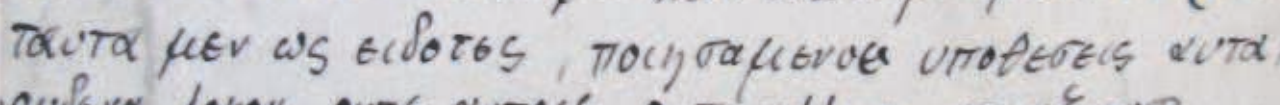
ouvera lopor oute autois outeallocs ETL a klovor

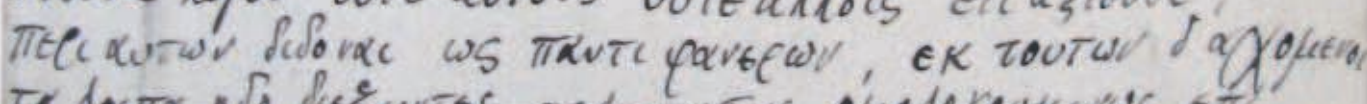

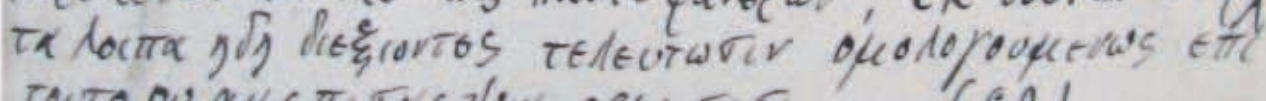
recto ov ave

of. arate of la tquilicie

ef. it atimus.

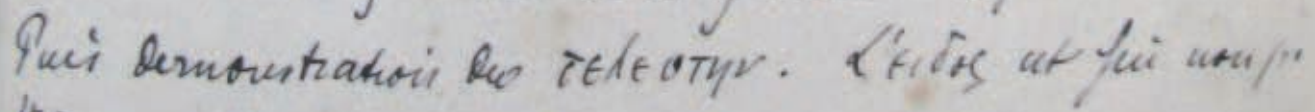

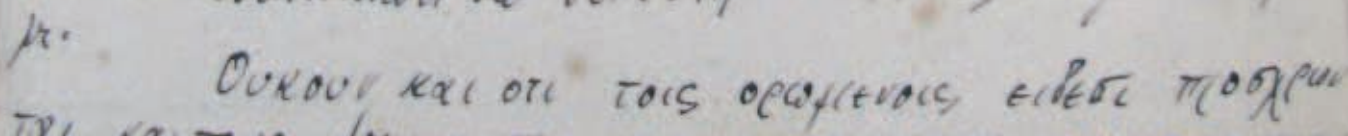

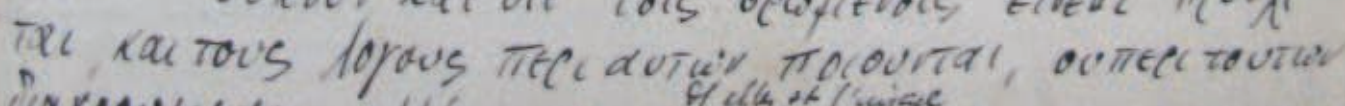

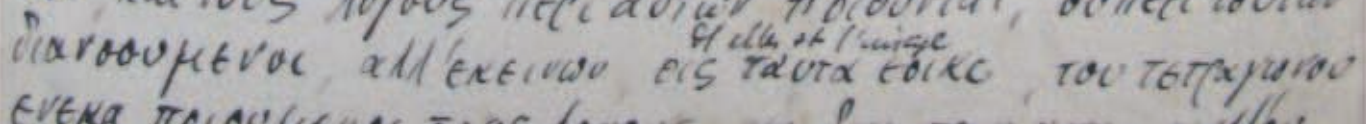

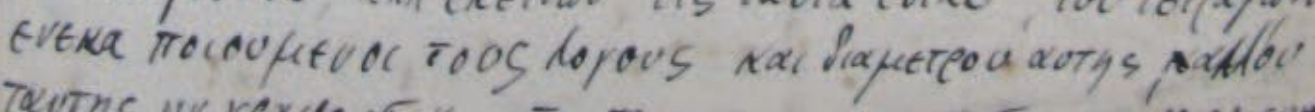

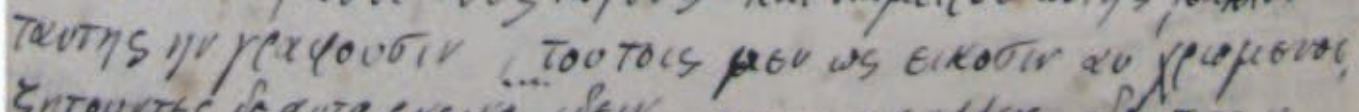

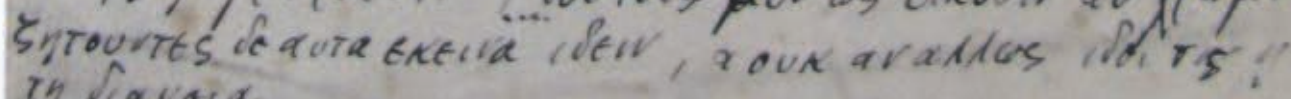
in fiavola.

Fiche de Marcel Mauss, s. d. (Fonds Mauss-IMEC)

20 


\section{Pour une archéologie du travail savant : fiches, livres et lettres de Marcel Mauss.}

$\mathrm{D}$

epuis plusieurs années, l'effort de conservation concernant les archives de la recherche en sciences humaines et sociales s'est principalement porté sur les données institutionnelles et quantitatives. Les archives personnelles des chercheurs, dans un certain sens plus qualitatives, posent des problèmes juridiques qu'il est encore difficile de résoudre à partir de la seule loi de 1979, qui précise que tout document produit dans le cadre d'une activité publique - par exemple par un chercheur dans un laboratoire - relève du domaine public et doit donc être considéré comme une archive publique ${ }^{1}$. Peut-on et doit-on tout conserver ? Quelles sont les institutions qui ont cette mission ? Mais ces archives de chercheurs posent également un problème scientifique puisqu'il s'agit de déterminer dans quelles conditions et selon quelles règles ces " données " peuvent constituer des sources disponibles pour de nouvelles recherches.

Ces archives ne sont en aucun cas de simples résidus, ni des restes insignifiants de l'activité scientifique. Au contraire, elles en constituent sans doute l'élément le plus marquant. La plupart du temps, elles permettent de suivre l'activité savante en train de se faire, une pensée, un savoir en train de se construire. Aussi, décider de prendre la " table de travail " du chercheur comme lieu d'enquête et d'analyse - ce qui nécessite d'identifier, de relever et de classer un grand nombre d'éléments, souvent minuscules - permet de porter le regard sur des pratiques savantes quotidiennes que l'on peut définir comme l'ensemble des opérations manuelles, discursives et intellectuelles mobilisées dans la production, la diffusion, la réception et l'usage des savoirs.
C'est dans cette perspective que nous avons voulu revisiter les différents fonds d'archives de Marcel Mauss que l'on a coutume de considérer aujourd'hui comme le père fondateur de l'ethnologie française. Ses archives (IMEC ${ }^{2}, \mathrm{MNHN}^{3}$, Quai Branly) renvoient à des activités scientifiques, administratives et pédagogiques mais aussi politiques. Pour autant, et malgré les publications de plus en plus nombreuses autour de ce " personnage " central de l'histoire des sciences humaines ${ }^{4}$, jamais ces documents n'ont été analysés pour ce qu'ils sont vraiment, à savoir des archives de la recherche en acte. Ces archives pourtant nous renseignent sur des pratiques, des rites (comme par exemple son entrée en 1930 au Collège de France), mais aussi sur les formes de savoir qui étaient pratiquées à son époque. Elles montrent que la recherche est un travail quotidien, une routine qui impose au savant différentes opérations, simples ou complexes, comme lire un livre, aller en bibliothèque, rédiger un compte rendu, prendre des notes, conserver les conclusions d'une discussion, décrire un phénomène, dessiner un objet, cartographier un lieu, écrire sur d'autres livres, répondre à des objections, défendre des hypothèses ${ }^{5}$...

Travailler à partir de l'archive - et prendre en compte sérieusement sa matérialité- n'est pas sans poser problème. D'ailleurs, les logiques de conservation sont bien loin de favoriser ce type de questionnement historique et épistémologique. Dans bien des cas, la correspondance qui a été classée par l'auteur lui-même est extraite et réorganisée selon un ordre alphabétique. Lorsque, par chance, une bibliothèque est conservée dans son intégralité, aucune information n'a véritablement été collectée sur le classement décidé ou subi par le propriétaire ${ }^{6}$ et comme pour 
les correspondances, les livres sont reclassés alphabétiquement puis dispersés dans les différents rayonnages, mélangés à d'autres ouvrages. La question des inserts est certainement la plus représentative de cette situation. Trop souvent, les feuilles griffonnées par les propriétaires des livres sont encore extraites par des bibliothécaires trop zélés pour qui un imprimé ne peut pas être, en même temps, une archive ! Ces situations sont d'autant plus difficiles à contourner que les inventaires sont perçus comme définitifs. La cumulativité des informations concernant un fonds est loin d'être une pratique courante, même lorsqu'il est confié à de grandes institutions d'archives comme l'IMEC.

Néanmoins, ce qui fait l'intérêt des archives de Marcel Mauss, c'est qu'elles renvoient à un moment tout à fait particulier de l'essor des sciences sociales qui, à la fin $\mathrm{du} 19^{\mathrm{e}}$ siècle, connaissent un changement d'échelle sans précédent, lui-même provoqué par l'invention et la diffusion des machines à écrire et du document dactylographié. Cette révolution dans les techniques documentaires inaugure un système inédit de traitement de l'information qui a suivi de près les multiples changements de la société elle-même (industrialisation, urbanisation, technicisation) ${ }^{7}$. C'est aussi durant cette période de la III ${ }^{e}$ République que les savants ont pour la première fois pu bénéficier d'une plus large audience dans la société en s'imposant face aux juristes et aux écrivains.

Je voudrais m'attacher plus particulièrement à trois objets qui, dans le cas de Mauss, font apparaître ces pratiques ordinaires de chercheur, d'autres lieux du savoir et, je l'espère aussi, l'image d'un monde savant beaucoup plus contrasté que ce qu'on a l'habitude d'en dire. Je voudrais tout d'abord revenir sur l'usage des fiches et l'enjeu de la classification des savoirs. Ensuite, le dispositif de la bibliothèque me permettra d'observer la manière dont Mauss utilise le livre dans son travail ethnographique. Enfin, je reviendrai sur un exemple particulier de l'épistolarité savante, les lettres de Mauss durant sa longue mobilisation sur le front, entre 1914 et 1918.

\section{Mettre le savoir en fiches.}

C'est durant ses études de philosophie à Bordeaux, entre 1891 et 1896 , que Mauss commence à pratiquer régulièrement la mise en fiches, témoignage direct de son intense activité intellectuelle. On découvre dans ses archives conservées plusieurs centaines de fiches de lecture et d'exercices répétés de rédaction où l'étudiant s'exerce à traiter de sujets aussi divers que "d'ou vient la croyance ? ", " le moi est-il une collection d'états de conscience ? ", " la mémoire ", ou encore " l'association mentale ". À cela s'ajoute aussi plusieurs notes théoriques, d'une certaine longueur, sur la photographie du mouvement, le problème de l'odorat, ou encore l'histoire du théâtre au Moyen Age. Mauss rédige également sur ses fiches plusieurs longs résumés d'œuvres philosophiques, comme les Regulae de Descartes, et construit des biographies de Malebranche, Gassendi, Leibniz ou Spinoza, tout en s'essayant à des tentatives de formalisation théorique sur la question des causes occasionnelles ou sur le spiritualisme.

Ces fiches sont hétérogènes, aussi bien sur le fond que dans leur forme. Elles vont du simple bout de papier déchiré à la récupération de ses fiches d'emprunt de la bibliothèque universitaire de Bordeaux. Il n'existe pas de format défini, ni de style bien reconnaissable. Chaque fiche est un document unique.

Quelques années plus tard, à l'occasion d'une recherche que Mauss mène aux côtés de l'historien des religions et folkloriste Robert Hertz, à la British Library, les fiches reflètent un nouvel enjeu méthodologique et épistémologique. En effet, Robert Hertz pratique assidûment la mise en fiche, qu'il conçoit comme la base de tout travail comparatif en histoire des religions : " Un bon répertoire bibliographique, la critique des textes que nous avons, en somme, apprise (c'est le seul profit de notre longue éducation classique), un système de travail par fiches pratique, rapide et méthodique, et l'on vient à bout, ce me semble, de la masse d'abord effroyable des faits $"{ }^{8}$. La fiche acquiert une qualité essentielle pour la plupart de ces ethnologues de " cabinet " de la fin du $19^{\mathrm{e}}$ siècle en leur permettant de s'orienter dans l'ensemble des traces documentaires accumulées par une activité de recherche.

Il serait faux cependant de croire que ces nouvelles possibilités - combinatoires, cumulatives, comparatives - de savoir liées à la technologie du fichier soient infinies.

Le meuble à fiches articulées, invention de la fin du $19^{\mathrm{e}}$ siècle, fixe un classement et ne permet que très rarement d'actualiser ou de modifier le contenu des fiches. Il y a aussi un effet pervers spécifique à cette 


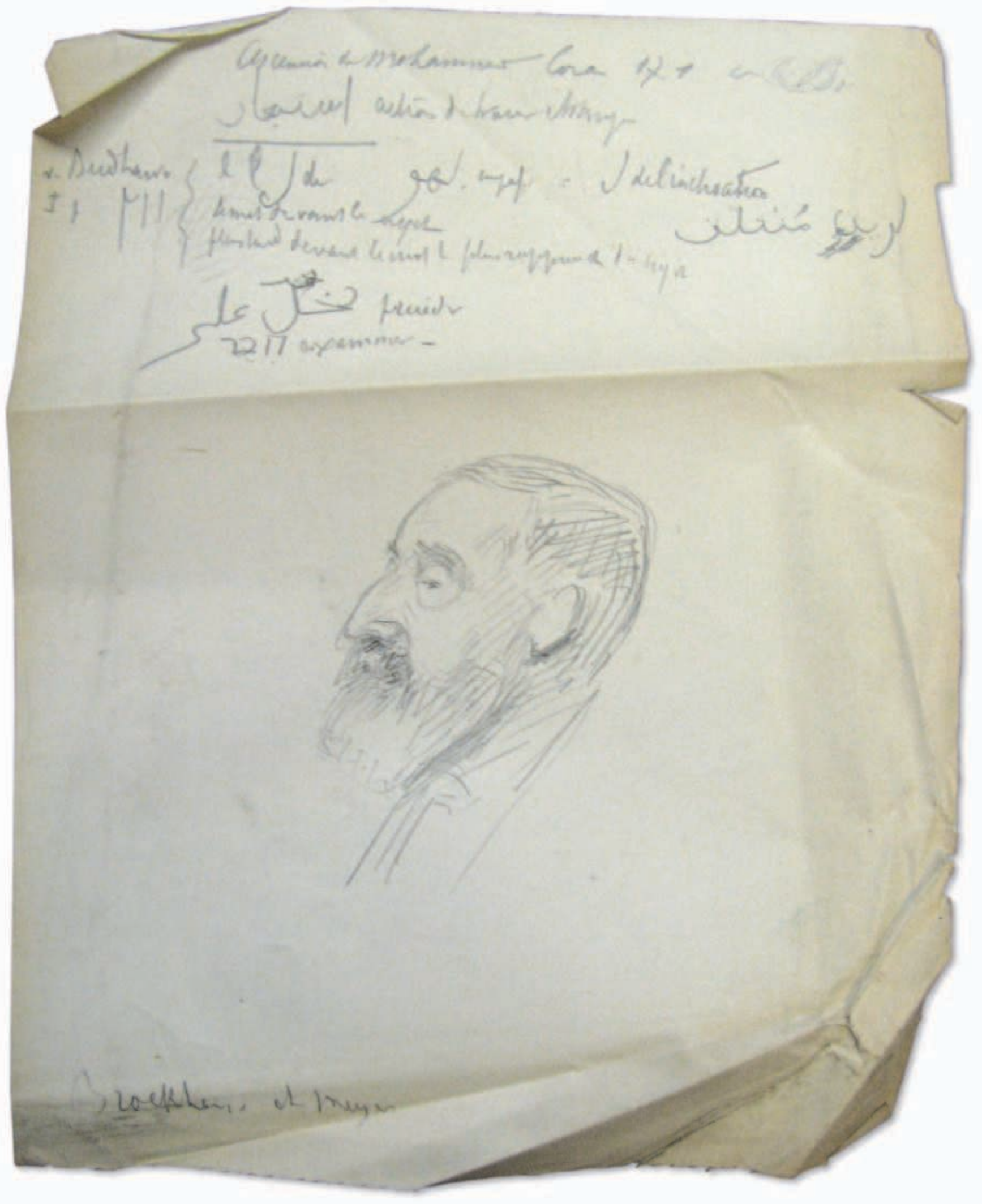

Insert découvert dans l'un des livres de Marcel Mauss (médiathèque du musée du quai Branly) 
opération de classification à grande échelle : le fichier rend l'acte de penser inconfortable. Parfois même, il devient un obstacle à la rédaction, à la formalisation, à la théorisation. Il donne la nausée - un sentiment qui est lié, d'abord, à la dimension routinière de ce travail qui consiste à rédiger quotidiennement des dizaines de fiches sans savoir exactement à quoi celles-ci pourront servir par la suite.

Afin d'éviter cet écueil, ces mêmes savants vont imaginer plusieurs palliatifs. Le premier consiste à créer un environnement de travail favorable. Le second vise à instaurer une distance avec le quotidien de la recherche - par exemple en allant travailler à la campagne. On retrouve ici la hantise d'une sédentarité mal dominée, où l'intellectuel passe le plus clair de son temps à sa table de travail, sacrifiant son corps au profit de la quête de la connaissance. Ces deux précautions sont jugées comme impératives pour respecter l'équilibre tant psychologique que physique $^{9}$. Mais elles posent un nouveau problème : comment travailler sérieusement à la campagne sans ses fiches, ses livres, ses notes ? La fiche et le livre sont des médiateurs du savoir. En cela, ils doivent être à portée de main.

\section{Que nous apprend une bibliothèque sur l'activité d'un chercheur?}

La bibliothèque d'un savant participe de cette mosaïque d'archives du travail scientifique. Elle montre elle aussi des pratiques ordinaires et banales qui souvent échappent au regard de l'historien. Du choix des sources aux techniques de lecture et au découpage des textes par des surlignements, des techniques de citation aux rapports entre bibliographies " primaire " et " secondaire ", la bibliothèque de Mauss nous donne un point de vue incomparable sur le cheminement de sa pensée et la construction de ses principaux concepts.

Cette bibliothèque, où se côtoient le " primitif ", le préhistorique, l'antique, le biologique, mais aussi le linguistique et le technique, témoigne d'un ensemble de séquences de travail, de séries de gestes qu'une ethnographie rétrospective est en mesure d'appréhender dès lors qu'elle se focalise sur les marques de lecture laissées par Mauss (signes, annotations, marginalia, mais aussi inserts) - des marques qui rendent compte de ses manières de lire et donc de travailler et de penser ${ }^{10}$. Une première observation concerne ses pratiques d'annotation. En effet, Mauss décide de placer ses notes en fonction de la physionomie générale du livre. Si les couvertures le permettent, c'est sur les rabats des jaquettes qu'il écrit. Parfois, c'est sur la quatrième de couverture qu'il inscrit ses remarques au crayon de papier. Ses interventions sont courtes. Il se contente le plus souvent de souligner par un trait de couleur (bleu ou rouge) les passages qu'il juge importants. Dans quelques cas, il encadre certains paragraphes. Parfois, il ajoute quelques mots en face du texte imprimé dans la couleur qu'il vient d'utiliser pour souligner la phrase ou le paragraphe. Ces notes sont la marque d'une lecture attentive, critique, qui donne la réplique aux auteurs par des corrections et des révisions. Elles sont aussi la marque de l'instantanéité d'une lecture, de l'envie d'adhérer ou au contraire de se distinguer d'une analyse, d'une observation ou d'une hypothèse.

De nombreuses annotations concernent les travaux des missionnaires, explorateurs ou administrateurs des colonies qui sont pour Mauss les premiers responsables d'observations, malheureusement imprécises et donc inutilisables par le sociologue et l'ethnologue.

Le livre du pasteur protestant Fernand Grébert (18861969) intitulé Au Gabon, découlant pourtant de plus de vingt ans d'activité missionnaire ${ }^{11}$, en fait largement les frais. Les annotations montrent une réaction quasi " épidermique " de Mauss devant les approximations ethnographiques de Grébert. Le texte de ce dernier, bien que tourné vers la description de la vie quotidienne et la culture matérielle des populations ${ }^{12}$ du Gabon, touche un point sensible pour Mauss qui enseigne au même moment à l'Institut d'ethnologie et à l'Ecole pratique des hautes études que l'observation doit être précise et sans jugement de valeur.

Parlant de l'alcoolisme, l'emploi du terme " eau de feu " par Grébert, en référence à la manière dont les Indiens d'Amérique définissaient cette boisson importée par les "Blancs ", fait réagir Mauss une première fois : "Vous vous croyez dans les Rocheuses " (p. 114). Quelques pages plus loin, lorsque le missionnaire évoque les relations de couple et surtout la " psychologie " de la femme gabonaise qui a encore, dans bien des cas, " un cœur de païenne ", Mauss ne peut s'empêcher d'ajouter : "Quelle rigolade " (p. 123). Lorsque Grébert insiste aussi sur les bienfaits de la conversion, Mauss s'exaspère et dans un geste rapide, inscrit : "Gardez-le pour le catéchisme " (p. 125 ; voir illustration p. 23). Il finira par " exécuter " l'auteur par 
si "bien" et que Nzé a voulu tuer par jalousie, et en méme temps elle se dit : "Pour ravoir mon enfant, je n'ai qu'à épouser Etughe. " Et elle envoie un gamin porter un paquet de nourriture avec un bracelet ou une bague à Etughe. Celui-ci, tout joyeux de ce muet appel, se munit de marchandises, de la fille de Nyingon et d'un cadeau pour le père, il apporte le tout à Na et devient l'èpoux de Nyingon; chacun est satisfait, le père, le gendre, la fille et son enfant.

Nzé arrive chez son beau-père : "Donne-moi ma femme, rends-moi mon enfant ! " L'ancien beau-père de répondre : "Oublies-tu que Nyingon avait le droit de te quitter et moi de la donner a Etughe ? Va-t'en, qu'ai-je encore à faire avec toi; je vais te rendre les quelques misérables marchandises que tu m'as données avec regret. " $\mathrm{Na}$ va chercher quelques-unes des marchandises qu'Etughe venait de lui apporter, car celles de Nzé étaient déjà depuis longtemps empruntées par d'autres, ou passées en paiement de préts ou de dettes, etc. Nzé réclame : "Ce n'est pas assez, je ne vois que la moitié de ce que tu me clois. ""Soit, dit Na, tu viendras les chercher plus tard. "-

En effet, si Nzé tient à se chercher femme une troisième fois, il lui faudra compléter son bien en allant sans cesse réclamer à son ancien beau-père ce que celui-ci lui doit encore. A son tour, il deviendra envers cet homme une espèce de davier, d'appareil extracteur, en ayant peut-ètre, en plus, le crève-cocur de revoir sa femme et son enfant, et de se trouver, devant la face narquoise d'Etughe, dans la position d'un mendiant qui menace un plus fort que lui. II ne faut pas s'étonner, que dans cez conditions de vie de famille, les Pahouins aient mangé leurs ennemis pour savourer leur vengeance. Chaque Pahouin peut dire qu'il a une ou plusieurs histoires semblables dans sa vie. Que peut ètre la vie d'un polygame dont les femmes sont rongèes de jalousie entre elles, et qu'il doit toutes maintenir pour lui seul! Quel miracle quand un Pahouin arrive à sortir de cet enfer pour se convertir! et l'on se demande à quel point les cœurs des païens sont endureis pour ne pas désirer en sortir en masse, par centaines.

La dot. - Le père pahouin reçoit des marchandises à la place de ses filles. Laissera-t-il les étoffes se ronger de mites, les matchettes se couvrir de rouille, la poudre se moisir? Il cherchera à placer toutes

Commentaires de Mauss sur le livre de F. Grébert, Au Gabon : Afrique équatoriale française, Société des missions évangéliques, 1928 (médiathèque du musée du quai Branly) 
un " Triste Grébert " (p. 151) suite à une longue description, bien peu ethnographique il est vrai, de l'expression de la tristesse au Gabon, un sujet que Mauss connaît bien pour l'avoir abordé pour la Mélanésie et l'Australie ${ }^{13}$.

\section{Lettres de guerre, lettres de savant.}

Les correspondances, comme les bibliothèques, sont de formidables révélateurs des positionnements sociaux et professionnels des savants. A qui écrivent-ils, et surtout comment ? Il existe des codes, bien souvent imperceptibles, mais qui nous renseignent sur la structuration et la hiérarchisation des milieux savants (éditeurs, collègues, étudiants...). Les lettres donnent encore l'image d'une science extrêmement vivante et mouvementée, faite d' " histoires ", de polémiques, de soutiens ou de défections...

L'analyse précise des correspondances conservées dans le fonds Mauss apporterait à n'en pas douter un éclairage tout à fait nouveau sur le processus de création et d'écriture au sein du groupe de L'Année sociologique, en particulier durant la Première Guerre mondiale. La longue mobilisation de Mauss sur le front en tant qu'interprète est l'un de ces moments rares où il fut confronté à de nouvelles perceptions de son entourage comme de son environnement, sans pour autant oublier son travail savant ${ }^{14}$.

Sa correspondance avec le linguiste Antoine Meillet (206 lettres entre 1914 et $1918^{15}$ ) est sur ce point extrêmement enrichissante. En effet, Meillet s'emploie à transmettre à Mauss les informations qu'il a pu glaner dans d'autres correspondances, en particulier avec ses contacts russes des Balkans. Durant les quatre années de guerre, il détaille les dernières nouvelles du front, signale les mouvements de troupes, fait l'hypothèse de l'entrée en guerre de la Roumanie en juillet $1916^{16}$. Il commente longuement les législations de guerre et les problèmes économiques qui en découlent. Meillet est alors en contact régulier avec Sylvain Lévi, Emile Durkheim et Lucien Lévy-Bruhl, ainsi qu'avec les linguistes Marcel Cohen et Joseph Vendryes. C'est lui, par exemple, qui prévient Mauss de la mort d'André Durkheim, comme de celle de Raoul Bloch le 22 mai 1916, ou de celle de Georges Boyer. Meillet se fait d'ailleurs de plus en plus de souci pour Mauss : " on a l'impression ici que vous allez écoper à votre tour ${ }^{17}$. Mais le linguiste profite de sa correspondance pour indiquer l'avancée de son travail. En mai 1916, il termine sa lettre en disant avoir travaillé " un peu " sur une petite note technique. Le 6 juillet, il ajoute : " je commence à mettre au point mon gros livre slave qui a besoin, je le vois, d'une sérieuse révision. Il est bon qu'un livre attende un peu (...) C'est une manière de préparer l'après-guerre ${ }^{18}$.

La guerre, la mobilisation des uns et des autres, n'empêchent pas le travail intellectuel. On sait, par exemple, que Mauss ne laisse pas totalement tomber ses lectures " érudites ", même si la situation ne s'y prête pas, surtout techniquement : " pour le Heine, j'attendrai puisqu'il est trop gros, d'être à ma permission. A ce moment-là je pourrai avoir un mobilier et une bibliothèque avec moi $"{ }^{19}$. D'autres lectures vont pour un temps lui importer beaucoup plus, comme celle des règlements d'infanterie qui doivent lui permettre de pallier son manque d'expérience sur le terrain. Ce qui est le plus précieux, écrit-il à Hubert, ce " sont les livres de tactique $"{ }^{20}$.

Si Mauss continue de travailler - comme si de rien n'était - son image changera dès 1916 pour ses amis proches. Paul Fauconnet ne se fait aucune illusion et lui indique comment cette guerre aura pour principale conséquence d'établir une différence nette entre ceux qui auront été au front, et ceux qui n'auront connu que les arrières : « Non seulement tu auras dans l'avenir le prestige de ceux qui sont allés au front, mais tu auras participé à la vie collective de l'armée et recueilli directement l'image de la guerre. Pour un moraliste et sociologue, c'est un enrichissement d'expérience qui n'est pas négligeable ${ }^{21}$.

Toutes les informations disséminées dans les correspondances témoignent d'un ensemble de pratiques et de séquences de travail qu'il n'est pas possible de comprendre autrement. Les lettres, les fiches, les bibliothèques sont chacune à leur manière des lieux privilégiés où se trouve reflété le travail de la recherche dans ses multiples dimensions. Elles montrent les investigations tâtonnantes, le quotidien, la part du travail documentaire, la routine de l'écriture, mais aussi les difficultés "logistiques " d'une recherche, les retards dus à la fatigue, aux problèmes de santé, à la guerre. Ces archives nous introduisent au cœur de l'atelier de la recherche en sciences sociales et éclairent la genèse des livres qui sont devenus des " classiques ". On suit les étapes de la pensée, la maturation des idées, la collecte et l'interprétation des données, à travers les dif- 
férentes traces matérielles qu'elles ont laissées. Cette genèse, ce contexte offrent à l'historien un point de vue privilégié sur la pratique même des savoirs, dans leurs dimensions matérielle, discursive, intellectuelle et sociale ${ }^{22}$.

\section{Jean-François Bert}

\section{Notes}

1 - Charmasson, Thérèse, Les archives des scientifiques XVI ${ }^{e}-X X^{e}$ siècle. Guide des fonds conservés en France. Paris : Editions du CTHS, 2008

2 - IMEC : Institut Mémoires de l'édition contemporaine

3- MNHM : Museum national d'Histoire naturelle

4 - Fournier, Marcel, Marcel Mauss. Paris : Fayard, 1994

5 - Françoise Waquet ajoute d'autres exemples qu'elle tire de son observation du monde universitaire : rédiger des remerciements, offrir des mélanges, faire une leçon inaugurale, écrire un portrait de savant dans un livre qui lui est consacré. Ces rituels révèlent, pour l'historienne, " dans leur diversité et leur complexité, les liens qui unissent les personnes, qui dessinent des communautés petites et grandes, qui composent des réseaux : les amis, collègues et élèves du dédicataire des mélanges ; le triangle formé par l'auteur, le bénéficiaire et le destinataire d'une recommandation ; la relation duale établie par la lettre échangée entre deux savants... ". Waquet, Françoise, Respublica academica, rituels universitaires et genres du savoir (XVII $-X X I^{e}$ siècles). Paris : PUPS, p. 21

6 - Il ne faut jamais sous-estimer les contraintes matérielles liées au manque de place, aux mobiliers utilisés, mais aussi aux déménagements successifs qui s'expliquent par les carrières internationales.

7 - Gardey, Delphine, Ecrire, calculer, classer. Comment une révolution de papier a transformé les sociétés contemporaines (1800-1940). Paris : La Découverte, 2008

8 - Lettre de Robert Hertz à Pierre Roussel datée du 21/03/1905, FRH $06 \mathrm{C} 03.001$ à 023

9 - Masseau, Didier, L'invention de l'intellectuel dans l'Europe du XVIII' siècle. Paris : PUF, 1994, en particulier p. 135-147

10 - Nous remercions Anne Faure, Frédérique Servain-Riviale et Ghislain Dibie du musée du quai Branly pour avoir rendu possible cette observation minutieuse des livres de Marcel Mauss.

11 - F. Grébert avait rejoint Albert Schweitzer à Lambaréné, c'est là qu'il apprit à parler le pahouin.

12 - Grébert collecta de nombreux objets qu'il envoya aux musées d'ethnographie de Genève et de Neuchâtel. Un ouvrage récent a été l'occasion de reprendre l'itinéraire de Grébert au Gabon : Le Gabon de Fernand Grébert, 1913-1932. Genève : Musée d'ethnographie / Éditions D, 2003.

13 - Mauss, Marcel, L'expression obligatoire des sentiments, in Euvres 3. Paris : Minuit

14 - Comme le note Christophe Prochasson concernant Robert Hertz décédé en 1915 : « la guerre renouvelle toujours le stock disponible des expériences humaines (...) plus généralement, les rapports à autrui connaissent un nouveau cours ". Prochasson, $\mathrm{Ch}$., Culture de guerre et consentement, préface à Un ethnologue dans les tranchées. Lettres de Robert Hertz à sa femme Alice. Paris : CNRS éditions, 2002, p. 27-34
15 - Cette correspondance montre aussi comment évoluent, sur plus de trente ans, les méthodes et les objets " communs » de la linguistique et de l'anthropologie comme, par exemple, la question des emprunts, le rapport entre langage et outillage mental, la question du bilinguisme ou celle de l'imposition d'un vocabulaire de culture, le rapport entre types d'écritures et types de sociétés, le problème de l'immanence de la langue.

16 - Ce n'est que le 27 août 1916 que la Roumanie déclarera la guerre à l'Autriche-Hongrie et à l'Allemagne. Au bout de trois mois, elle sera défaite militairement.

17 - Lettre de Meillet à Mauss, 5 juin 1916. Fonds Mauss, IMEC

18 - Lettre de Meillet à Mauss, 6 juillet 1916. Fonds Mauss, IMEC

19 - Lettre de Mauss à Hubert, 30 mars 1916. Fonds Mauss, IMEC

20 - Lettre de Mauss à Hubert, 7 mars 1917. Fonds Mauss, IMEC

21 - Fauconnet a connu durant la guerre une vie administrative. Il se lamentera de cette expérience minimale : «j'espère que la ze année de guerre sera moins stérile que la seconde. En lectures, en notes, en idées, j'ai accumulé déjà, surtout depuis que je suis à [ill.], pas mal d'éléments des travaux sur le socialisme qui m'occuperont si... et quand... ». Lettre de Paul Fauconnet à Marcel Mauss, s.d. Fonds Mauss, IMEC

22 - Pour en savoir plus sur la manière d'appréhender ses archives, voir Bert, Jean-François, Dansl'atelier de Marcel Mauss. Paris : CNRS éditions, 2012

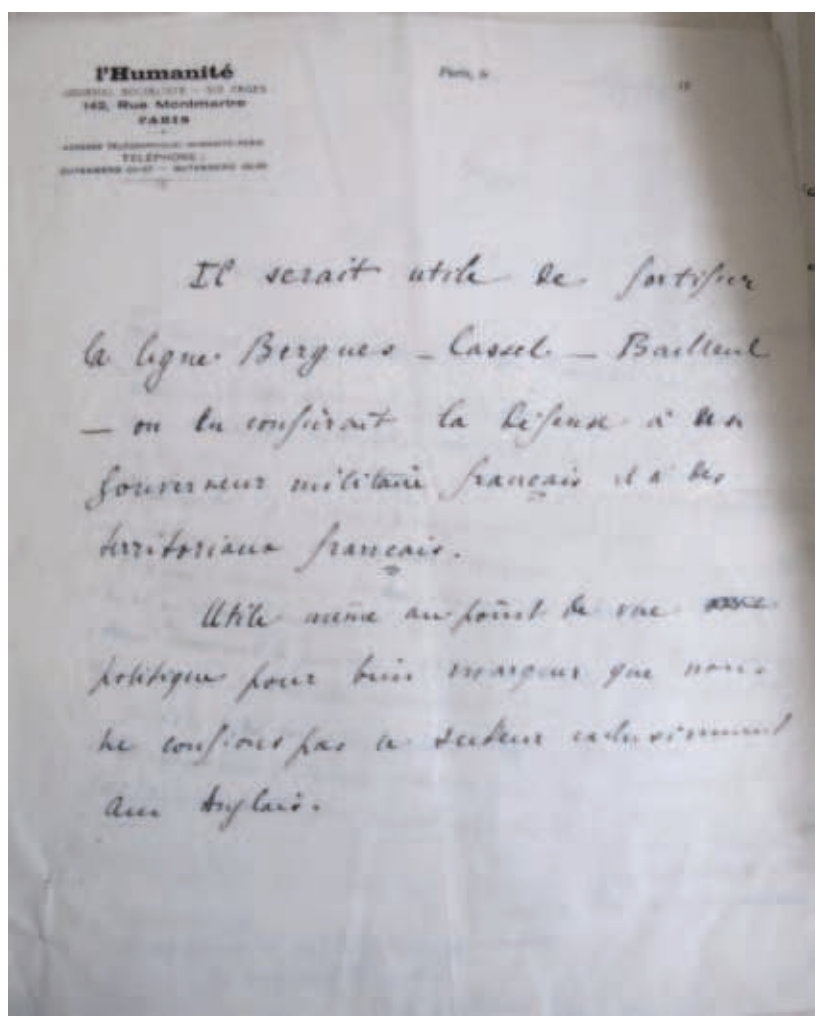

Commentaires de Marcel Mauss concernant les fortifications militaires durant la Première Guerre mondiale, sur un papier à en-tête du journal L'Humanité, s. d. (Fonds Mauss, IMEC) 